\title{
Closing Patent Foramen Ovale (PFO) for Cryptogenic Stroke - Deeper Thoughts
}

\section{Cheuk-Kit Wong *}

Department of Medicine and Therapeutics, Faculty of Medicine, The Chinese University of Hong Kong, Hong Kong

${ }^{*}$ Corresponding author: Cheuk-Kit Wong, MD, FRCP, FRACP, FACC, Department of Medicine and Therapeutics, Faculty of Medicine, The Chinese University of Hong Kong, Prince of Wales Hospital Shatin, Hong Kong, Tel: 852-35053139; E-mail: cheuk-kit.wong@cuhk.edu.hk

Received date: March 16, 2018; Accepted date: March 26, 2018; Published date: March 30, 2018

Copyright: (c) 2018 Wong CK. This is an open-access article distributed under the terms of the Creative Commons Attribution License, which permits unrestricted use, distribution, and reproduction in any medium, provided the original author and source are credited.

\section{Editorial}

In the fetal heart, the foramen ovale (open channel between the septum primum and the septum secundum) allows oxygenated blood returning from the umbilical vein to pass into the left heart and be pumped into the systemic circulation. The Eustachian valve between the inferior vena cava and the right atrium directs blood flow through the foramen ovale into the left atrium.

The foramen ovale remains patent in up to a quarter of adults thus traditionally considered a normal variation. However, patent foramen ovale (PFO) is found to be more prevalent among patients having cryptogenic stroke in whom the mechanism for the ischemic stroke is not found despite an extensive evaluation of head and neck vessels and the heart. Paradoxical embolism across the PFO likely explains the stroke.

\section{The PFO Closure Trials for Cryptogenic Stroke}

To support this theory of paradoxical embolism closing PFO should reduce recurrence of ischemic stroke. The development of closure devices delivered through a trans-catheter route (without open-heart surgery) allows PFO closure to be performed safely with low morbidities. However, three randomized trials reported early in this decade (CLOSURE I [1], PC trial [2], and RESPECT [3]) individually failed to show a clear benefit of trans-catheter PFO device closure for secondary stroke prevention. In retrospect, this is partly due to the individually small-size cohorts and partly the short follow-up durations. More recently, a meta-analysis of patient-level data from CLOSURE I, PC, and RESPECT (with longer follow-up duration) found that PFO closure prevented recurrent ischemic stroke (adjusted hazard ratio [HR], 0.58; 95\% confidence interval [CI], 0.34-0.99) [4]. Further, the Amplatzer PFO occluder device out-performed its counterparts, and received Food and Drug Administration (FDA) approval for patients aged 18-60 years with $\mathrm{PFO}$ and cryptogenic stroke.

Last year, two randomized controlled PFO closure trials (CLOSE [5] and REDUCE [6]) with stricter enrolment criteria were published. CLOSE included patients aged 16-60 years with a cryptogenic stroke supported by imaging findings within the prior 6 months and a PFO with large inter-atrial shunt or atrial septal aneurysm. CLOSE also used stringent criteria to exclude patients with vascular disease which may be potentially causal (intra-cerebral small vessel disease or $\geq 30 \%$ stenosis of an artery supplying the brain). Over 5.3 years of mean follow-up, the closure group had a lower risk of recurrent stroke than the non-closure group (0\% vs. $6 \%$; HR, $0.03 ; 95 \% \mathrm{CI}, 0-0.26$ ) [5].

REDUCE included patients aged 18-59 years with cryptogenic stroke (symptoms $\geq 24$ hours or positive imaging) in the prior 6 months and a PFO; but without any stenosis of $\geq 50 \%$ of a major vessel supplying the brain, lacunar lesions on imaging, or uncontrolled stroke risk factors. After a median follow-up of 3.2 years, there was a lower risk of recurrent stroke in patients with than in patients without PFO closure (1.4\% vs. 5.4\%; HR, 0.23; 95\% CI, 0.09-0.62) [6].

The stricter enrolment criteria in CLOSE and REDUCE likely enrolled mostly participants having stroke from paradoxical embolism while excluding those harbouring common "stroke mechanisms" such as large artery atherosclerosis, small vessel disease, or atrial fibrillation.

In March 2018, the Korean DEFENSE-PFO trial [7] was reported in a late-breaking trials session in the American College of Cardiology annual scientific meeting. This open-label trial included only cryptogenic stroke patients and used detailed transesophageal echocardiographic evaluation to identify those with high-risk PFO features including atrial septal aneurysm, hypermobility (septal excursion $\geq 10 \mathrm{~mm}$ during the cardiac cycle) or PFO size (maximum separation of the septum primum from the secundum) $\geq 2 \mathrm{~mm}$. Enrolment was stopped early after the positive PFO closure publications [4-6]. Medical therapy included anticoagulants or antiplatelet drugs according to the managing doctors but none received NOACs. The primary endpoint was a composite of stroke, vascular death or TIMI-defined major bleeding.

Of 450 patients having cryptogenic stroke and PFO, 175 had highrisk PFO features (38.9\%). Finally, 120 patients got randomized: 60 to PFO device closure plus medical therapy and 60 to medical therapy alone. All closure attempts were successful. Procedural complications included atrial fibrillation $(n=2)$, pericardial effusion $(n=1)$ and puncture site pseudoaneurysm $(n=1)$. The median duration of followup was 2.8 years (interquartile range, $0.9-4.1$ years). No patient in the device closure group had primary endpoint events during follow-up versus six patients in the medical therapy group $(p=0.013)$. These events included ischemic stroke $(n=5)$, cerebral hemorrhage $(n=1)$, TIMI-defined major bleeding $(\mathrm{n}=2)$, and transient ischemic attack $(\mathrm{n}=1)[7]$.

\section{The Missing Link Amidst Trans-Catheter PFO Closure and Stroke Prevention}

A pre-requisite for paradoxical embolism is clot formation in the venous system. However, clinically apparent deep vein thrombosis (DVT) or pulmonary embolism (PE) are infrequent among patients with cryptogenic stroke suggesting that venous clot formation has otherwise been sub-clinical.

While cryptogenic stroke is not rare, DVT/PE are more prevalent with theoretically up to a quarter of patients also having PFO. How often do these patients with DVT/PE end up with paradoxical 
embolism and ischemic stroke? High-risk anatomical features of PFOs include residual remnants of Eustachian valve directing blood flow to the PFO or atrial septal aneurysm enabling the patent PFO channel to open "better or wider" during the whole or part of the cardiac cycle. Valsalva or similar manoeuvres (even straining in toilet) produce the necessary physiologic oscillations of left and right atrial pressure during the "Valsalva respiratory cycle" for right-to-left inter-atrial shunting across PFO. These are the mechanistic basis for paradoxical embolism. Based on DEFENSE-PFO [7] about two-fifth of cryptogenic stroke patients have PFO had high-risk features. The percentage can be lower in patients without cryptogenic stroke, and lacking these highrisk features PFO may be completely benign.

Three questions now arise. First, should PFO closure be offered to patients with history of DVT / PE and also having PFOs with high-risk features? Second, should we target the venous clot formation by longterm anti-coagulation in patients with prior cryptogenic stroke and un-intervened PFOs? Third, should anti-coagulation be prolonged in DVT/PE patients who also have PFO (with more prolongation in those with more high-risk features).

Anti-coagulation with warfarin for a defined period has been the traditional therapy for DVT/PE. Two trials published early in this decade showed that with un-provoked DVT maintenance aspirin $100 \mathrm{mg}$ daily is superior to placebo after the initial warfarin therapy $[8,9]$. Although newer antiplatelet drugs may be better than aspirin in countering arterial thrombosis (a subject of several editorials in this journal [10-13]), venous thrombosis is predominantly driven by activation of clotting factors. Last year, the randomized Einstein CHOICE study [14], including 3365 patients who had finished the initial period of anticoagulation showed that long-term rivaroxaban 10 $\mathrm{mg}$ daily is superior to aspirin $100 \mathrm{mg}$ daily in preventing recurrent DVT / PE (hazard ratio 0.26, 95\% 0.14-0.47). While it should not be directly compared, this degree of risk reduction is similar to what has been shown in the PFO closure trials [4-7] using recurrent stroke as the end-point.

It remains unknown as to how PFO closure compares to systemic anticoagulation with NOACs for cryptogenic stroke. Are these patients actually also at risk for DVT / PE so that long-term anticoagulation may also represent primary prevention therapy? Along this path it should be noted that PFO device closure was associated with a higher risk of atrial fibrillation for which anticoagulation is often used. Without atrial fibrillation, patients are usually prescribed dual antiplatelet regimen (aspirin+clopidogrel) after device closure for at least 6 months.

$\mathrm{PFO}$ closure is not a complex interventional procedure but requires expertise, X-Ray fluoroscopy, operating team arrangement and a high one-off cost. NOAC therapy has bleeding risks even with reduced dosages and long-term therapy is expensive. However, evidence has come from the COMPASS trial of 27,395 patients with established stable atherosclerotic vascular disease [15], that rivaroxaban can also improve outcome of arterial disease.

The usual daily dose of rivaroxaban is $20 \mathrm{mg}$ daily. In COMPASS [15], a trial with 3-arm randomisation, rivaroxaban $5 \mathrm{mg}$ twice daily is comparable to aspirin $100 \mathrm{mg}$ in preventing the primary composite outcome of cardiovascular death, stroke, or myocardial infarction albeit with more serious bleeding complications. Rivaroxaban $2.5 \mathrm{mg}$ twice daily adding onto aspirin $100 \mathrm{mg}$ daily reduced the composite primary outcome $(24 \%$ relative reduction) without any significant increase in intracranial or fatal bleeding over aspirin $100 \mathrm{mg}$ daily alone. This superior outcome led to premature termination of the trial after a mean follow-up of only 23 months.

Apart from rivaroxaban, other widely used NOACs include dabigatran, apixaban and edoxaban. In previous PFO trials [1-7], the "mainstream" medical therapy in patients not undergoing PFO closure were mostly aspirin and sometimes warfarin. NOACs should now be the new comparators to PFO closure, testing both conventional and reduced dosages. In addition, very low dose NOAC (such as rivaroxaban $2.5 \mathrm{mg}$ twice daily) with concomitant aspirin or other antiplatelet agent may also be a meaningful comparator particularly when concomitant atherosclerotic vascular disease is present.

Paradoxical embolism causes not only cryptogenic stroke but also embolism in other arterial territories including the coronary circulation. This field is evolving. The good news is that we now have the full armamentarium to fight this disease by blocking coagulation activation, blocking platelet activation and blocking the culprit thrombus transition path through the PFO.

\section{References}

1. Furlan AJ, Reisman M, Massaro J (2012) CLOSURE I Investigators 2012 Closure or medical therapy for cryptogenic stroke with patent foramen ovale. N Engl J Med 366: 991-999.

2. Meier B, Kalesan B, Mattle HP (2013) PC Trial Investigators 2013 Percutaneous closure of patent foramen ovale in cryptogenic embolism. N Engl J Med 368: 1083-1091.

3. Carroll JD, Saver JL, Thaler DE (2013) RESPECT Investigators 2013 Closure of patent foramen ovale versus medical therapy after cryptogenic stroke. N Engl J Med 368: 1092-1100.

4. Kent DM, Dahabreh IJ, Ruthazer R (2016) Device closure of patent foramen ovale after stroke: pooled analysis of completed randomized trials. J Am Coll Cardiol 67: 907-917.

5. Mas JL, Derumeaux G, Guillon B (2017) CLOSE Investigators 2017 Patent foramen ovale closure or anticoagulation vs. antiplatelets after stroke. N Engl J Med 377: 1011-1021.

6. OpenUrlSøndergaard L, Kasner SE, Rhodes JF (2017) Gore REDUCE Clinical Study Investigators 2017 Patent foramen ovale closure or antiplatelet therapy for cryptogenic stroke. N Engl J Med 377: 1033-1042.

7. Lee PH, Song JK, Kim JS (2018) Cryptogenic Stroke and High-Risk Patent Foramen Ovale: The DEFENSE-PFO Trial. J Am Coll Cardiol.

8. Becattini C, Agnelli G, Schenone A (2012) WARFASA Investigators. Aspirin for preventing the recurrence of venous thromboembolism. N Engl J Med 366: 1959-1967.

9. Brighton TA, Eikelboom JW, Mann K (2012) ASPIRE Investigators. Lowdose aspirin for preventing recurrent venous thromboembolism. N Engl J Med 367: 1979-1987.

10. Wong CK (2012) Measuring Platelet Reactivity after Clopidogrel-Has it Reached the End of the Road. Cardiol Pharmacol 1: e105.

11. Wong CK (2016) From Arctic to Antarctic: Is There any Remaining Role for Platelet Function Testing to Tailor Therapy after Coronary Stent Implantation? Cardiovasc Pharm Open Access 5: e132.

12. Wong CK (2017) Trials Evaluating Ticagrelor in Cardiovascular Disease: Will It Reign Supreme in the Anti-Platelet World. Cardiovasc Pharm Open Access 6: e133.

13. Wong CK (2017) Tailoring Dual Antiplatelet Therapy after Coronary Stenting: The PRECISE-DAPT Score versus the DAPT Score. Cardiovasc Pharm Open Access 6: e135.

14. Weitz JI, Lensing AWA, Prins MH (2017) EINSTEIN CHOICE Investigators. Rivaroxaban or Aspirin for Extended Treatment of Venous Thromboembolism. N Engl J Med 376: 1211-1222.

15. Eikelboom JW, Connolly SJ, Bosch J (2017) COMPASS Investigators. Rivaroxaban with or without Aspirin in Stable Cardiovascular Disease. N Engl J Med 377: 1319-1330. 